\title{
On the Finiteness Problem for Automaton (Semi)groups
}

\author{
Ali Akhavi* $\quad$ Ines Klimann ${ }^{\dagger} \quad$ Sylvain Lombardy ${ }^{\ddagger} \quad$ Jean Mairesse $^{\dagger}$ \\ Matthieu Picantin ${ }^{\dagger}$
}

October 29, 2018

\begin{abstract}
This paper addresses a decision problem highlighted by Grigorchuk, Nekrashevich, and Sushchanskiü, namely the finiteness problem for automaton (semi)groups. For semigroups, we give an effective sufficient but not necessary condition for finiteness and, for groups, an effective necessary but not sufficient condition. The efficiency of the new criteria is demonstrated by testing all Mealy automata with small stateset and alphabet. Finally, for groups, we provide a necessary and sufficient condition that does not directly lead to a decision procedure.
\end{abstract}

\section{Introduction}

Automaton (semi)groups - short for semigroups generated by Mealy automata or groups generated by invertible Mealy automata - were formally introduced a half century ago (for details, see 9 and references therein). Two decades later, important results have started revealing their full potential. In particular, contributing to the Burnside problem, [1, 16] construct Mealy automata generating particularly simple infinite torsion groups, and, answering to the Milnor problem, 7, 17. produce Mealy automata generating the first examples of (semi)groups with intermediate growth.

Since these pioneering works, a substantial theory continues to develop using various methods, ranging from finite automata theory to geometric group theory, and various viewpoints from self-similarity to natural actions on regular rooted trees (see [5, 6, 8, 9, 18, 19, 24] for groups and $7,12,18,21,22,27$, for semigroups) and never ceases to show that automaton (semi)groups possess multiple interesting and sometimes unusual features.

The classical decision problems have been investigated for automaton groups and semigroups: the word problem is solvable 12, 18, while the conjugacy problem has recently been proved to be unsolvable 29. Here we address the finiteness problem, that is, the question of the existence of an algorithm that takes as input a Mealy automaton and decides if the generated (semi)group is finite (see [18, Problem 7.2.1(b)]). Since the word problem is solvable, then a semidecision procedure for the finiteness problem simply consists of enumerating all the elements.

Three results related to the finiteness problem have to be mentioned here. First, the finiteness problem is solved for the special class of semigroups generated by (dual) Cayley machines (see [12, 21, 22, 27]) by using semigroup theory and especially the Green's relations machinery. Second, the class of those automata which always generate finite (semi)groups independently of their output function has been completely characterized (see [2, 3, 25]). Third, the class of so-called "bounded" (invertible) automata where all the states have growth degree at most 0 has been thoroughly studied and the solution to the order problem (see [10, 26]) yields an infiniteness criterion. Observe that these three classes correspond to very special structures for the concerned Mealy automata.

\footnotetext{
*GREYC - CNRS UMR 6072 \& Université de Caen, France

${ }^{\dagger}$ LIAFA - CNRS UMR 7089 \& Université Paris Diderot-Paris 7, France

${ }^{\ddagger}$ LIGM - CNRS UMR 8049 \& Université Paris Est, France
} 
Two GAP packages are dedicated to automaton (semi)groups: FR by Bartholdi and automgrp by Muntyan and Savchuk [4, 13, 23]. Both include specific (in)finiteness tests. Besides the three results above-mentioned, all that was known up to now about the finiteness question for automaton groups happened to be somehow summarized in the documentation for FR:

\begin{abstract}
The order of [an automaton] group is computed as follows: if all [the states have growth degree at most 0], then enumeration will succeed in computing the order. If the action of the group is primitive, and it comes from a bireversible automaton, then the Thompson-Wielandt theorem is tested against [...] see [11, Prop. 2.1.1]. Then, FR attempts to find whether the group is level-transitive (in which case it would be infinite). Finally, it attempts to enumerate the group's elements, testing at the same time whether these elements have infinite order.
\end{abstract}

Needless to say, none except the first few steps are guaranteed to succeed.

In this paper, we give several new criteria for testing (in)finiteness, that could easily be added to the FR and automgrp packages. The original ingredients in these packages mainly come from geometric group theory. Our new notions and tools - like helix graphs and minimizationdualization - are automata-theoretic in nature and most often work in the general setting of semigroups. The common idea is to put a special emphasis on the dual automaton, obtained by exchanging the roles of stateset and alphabet. The stepping stone is Proposition 2.7 stating that any Mealy automaton generates a finite semigroup if and only if so does its dual. The general strategies vary by analyzing a Mealy automaton and its dual either alternatively - see the minimization-dualization reduction in Section 3 - or both together as a whole — see the helix graph construction in Section 4

In Section 3. we give an effective sufficient but not necessary condition for finiteness using minimization-dualization. Focusing on those invertible automata with invertible dual, and using helix graphs, Section 4 provides an effective necessary but not sufficient condition for finiteness, and also a non-effective necessary and sufficient condition. The decidability of the finiteness problem remains open.

Gathering the new criteria with the previously known ones allows to decide the (semi)group (in)finiteness for substantially more Mealy automata. In Table 1 we report on the results of the experimentation carried out on: $(i)$ all 3-letter 2-state Mealy automata; (ii) all 3-letter 3-state invertible or reversible Mealy automata. The first three columns are the number of automata treated successfully respectively by previously known criteria, our new criteria, and the union of both. The last column is the total number of Mealy automata. The automata are counted up to isomorphism.

Table 1: Some results of the experimentations to decide (in)finiteness with old and new criteria.

\begin{tabular}{|l|c|c|c|c|}
\cline { 2 - 5 } \multicolumn{1}{c|}{} & previous criteria & new criteria & previous+new & total \\
\hline general $(3,2)$ & 398 & 1130 & 1214 & 4003 \\
\hline inv. or rev. $(3,3)$ & 78721 & 100924 & 172737 & 236558 \\
\hline
\end{tabular}

More detailed experimental results are given in Section 5 and a gallery of meaningful examples is given in Table 2

\title{
2 Preliminaries
}

Let $S$ be a finite and non-empty set. We denote by $\mathfrak{T}_{S}$ the set of functions from $S$ to $S$, and we denote by $\mathfrak{S}_{S}$ the set of bijections from $S$ to $S$.

\section{$2.1 \quad$ Mealy automaton}

If one forgets about initial and final states, a (finite, deterministic and complete) automaton $\mathcal{A}$ is a triple $\left(A, \Sigma, \delta=\left(\delta_{i}: A \rightarrow A\right)_{i \in \Sigma}\right)$, where the set of states $A$ and the alphabet $\Sigma$ are non-empty 
finite sets, and where the $\delta_{i}$ 's are functions. In a condensed way, the automaton is identified with $\delta$, that is an element of $\mathfrak{T}_{A}^{\Sigma}$.

A Mealy automaton is a quadruple

$$
\left(A, \Sigma, \delta=\left(\delta_{i}: A \rightarrow A\right)_{i \in \Sigma}, \rho=\left(\rho_{x}: \Sigma \rightarrow \Sigma\right)_{x \in A}\right),
$$

such that both $(A, \Sigma, \delta)$ and $(\Sigma, A, \rho)$ are automata. Another standard terminology for Mealy automaton would be: letter-to-letter transducer with the same input and output alphabets. A Mealy automaton is identified with an element of $\mathfrak{T}_{A}^{\Sigma} \times \mathfrak{T}_{\Sigma}^{A}$.

Graphically, a Mealy automaton is represented by a labelled directed graph with:

$$
\text { nodes : } A, \quad \operatorname{arcs} \text { (transitions) }: x \stackrel{i \mid j}{\longrightarrow} y \text { if } \delta_{i}(x)=y \text { and } \rho_{x}(i)=j .
$$

The notation $x \stackrel{\mathbf{u} \mid \mathbf{v}}{\longrightarrow} y$ with $\mathbf{u}=u_{1} \cdots u_{n}, \mathbf{v}=v_{1} \cdots v_{n}$ is a shorthand for the existence of a path $x \stackrel{u_{1} \mid v_{1}}{\longrightarrow} x_{1} \stackrel{u_{2} \mid v_{2}}{\longrightarrow} x_{2} \cdots x_{n-1} \stackrel{u_{n} \mid v_{n}}{\longrightarrow} y$ in $\mathcal{A}$.

Two examples of Mealy automata are given in Fig. 1.
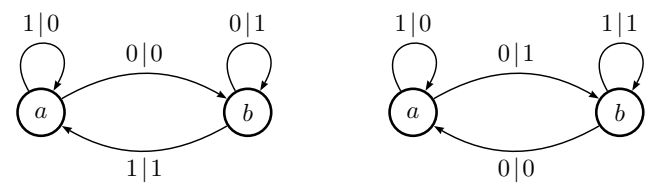

Figure 1: Two Mealy automata.

In a Mealy automaton $(A, \Sigma, \delta, \rho)$, the sets $A$ and $\Sigma$ play dual roles. So we may consider the dual (Mealy) automaton defined by $\mathfrak{d}(\mathcal{A})=(\Sigma, A, \rho, \delta)$. Alternatively, we can define the dual Mealy automaton via the set of its transitions:

$$
x \stackrel{i \mid j}{\longrightarrow} y \in \mathcal{A} \quad \Longleftrightarrow \quad i \stackrel{x \mid y}{\longrightarrow} j \in \mathfrak{d}(\mathcal{A}) .
$$

In what follows, it is often pertinent to consider a Mealy automaton and its dual together, that is to work with the pair $\{\mathcal{A}, \mathfrak{d}(\mathcal{A})\}$. A pair of dual Mealy automata is represented in Fig. 2 ,
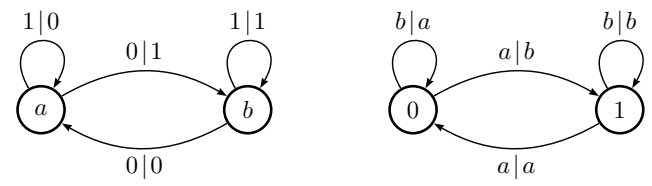

Figure 2: A pair of dual Mealy automata.

Consider a Mealy automaton $\mathcal{A} \in \mathfrak{T}_{A}^{\Sigma} \times \mathfrak{S}_{\Sigma}^{A}$. Let $A^{-1}=\left\{x^{-1}, x \in A\right\}$ be a disjoint copy of $A$. The inverse (Mealy) automaton $\mathcal{A}^{-1} \in \mathfrak{T}_{A^{-1}}^{\Sigma} \times \mathfrak{S}_{\Sigma}^{A^{-1}}$ is defined by the set of its transitions:

$$
x \stackrel{i \mid j}{\longrightarrow} y \in \mathcal{A} \quad \Longleftrightarrow \quad x^{-1} \stackrel{j \mid i}{\longrightarrow} y^{-1} \in \mathcal{A}^{-1} .
$$

Let us call respectively dualization (denoted $\mathfrak{d}$ ) and inversion (denoted $\mathfrak{i}$ ) the two transformations on transitions defined in (11) and (2). Starting with a transition and alternating the dualization and inversion transformations, we obtain eight transitions. (In the process, we also define $\Sigma^{-1}=\left\{x^{-1}, x \in \Sigma\right\}$, a disjoint copy of $\Sigma$; and we set $\left(A^{-1}\right)^{-1}=A$ and $\left(\Sigma^{-1}\right)^{-1}=\Sigma$.)

Now consider a Mealy automaton $\mathcal{A}$ identified with its set of transitions, and apply the same transformations to $\mathcal{A}$. We obtain eight sets of transitions that we denote by:

$$
\mathcal{A}, \mathfrak{d}(\mathcal{A}), \mathfrak{i}(\mathcal{A}), \mathfrak{d i}(\mathcal{A}), \mathfrak{i} \mathfrak{d}(\mathcal{A}), \mathfrak{d i d}(\mathcal{A}), \mathfrak{i d} \mathfrak{i}(\mathcal{A}), \operatorname{did} \mathfrak{i}(\mathcal{A})=\mathfrak{i d i d}(\mathcal{A}) .
$$

If $\mathcal{A} \in \mathfrak{T}_{A}^{\Sigma} \times \mathfrak{S}_{\Sigma}^{A}$, then $\mathfrak{i}(\mathcal{A})=\mathcal{A}^{-1}$. Apart from $\mathfrak{d}(\mathcal{A})$ which is always a Mealy automaton, the other six sets may or may not define a Mealy automaton depending on $\mathcal{A}$. 
By tracking the content of the sets of transitions, we observe the following:

$$
\begin{aligned}
{[\mathfrak{d i d i}(\mathcal{A}) \in} & \left.\mathfrak{T}_{A}^{\Sigma} \times \mathfrak{T}_{\Sigma}^{A}\right] \Longrightarrow \\
& {\left[\mathcal{A}, \mathfrak{d}(\mathcal{A}), \mathfrak{i}(\mathcal{A}), \mathfrak{d i}(\mathcal{A}), \mathfrak{i j}(\mathcal{A}), \mathfrak{d i d}(\mathcal{A}), \mathfrak{i j i}(\mathcal{A}), \mathfrak{d i d i}(\mathcal{A}) \in \mathfrak{S}_{A}^{\Sigma} \times \mathfrak{S}_{\Sigma}^{A}\right] . }
\end{aligned}
$$

Let us introduce some additional terminology.

Definition 2.1 A Mealy automaton is invertible if it belongs to $\mathfrak{T}_{A}^{\Sigma} \times \mathfrak{S}_{\Sigma}^{A}$; and reversible if it belongs to $\mathfrak{S}_{A}^{\Sigma} \times \mathfrak{T}_{\Sigma}^{A}$. A Mealy automaton is an IR-automaton if it is both invertible and reversible, that is, if it belongs to $\mathfrak{S}_{A}^{\Sigma} \times \mathfrak{S}_{\Sigma}^{A}$. If $\mathfrak{d i d i}(\mathcal{A})$ is a Mealy automaton, we say that $\mathcal{A}$ (resp. $\mathfrak{d}(\mathcal{A}), \ldots, \mathfrak{d i d i}(\mathcal{A}))$ is bireversible.

The terms "invertible, reversible, and bireversible" are standard since [20]. The acronym IRautomaton is introduced for convenience. IR-automata are of particular interest and the core of the paper is devoted to them. In Fig. 1, the right Mealy automaton is an IR-automaton, but not the left one.

Mealy automaton of order $(n, k)$. Consider a Mealy automaton $\mathcal{A}=(A, \Sigma, \delta, \rho)$ in $\mathfrak{T}_{A}^{\Sigma} \times \mathfrak{T}_{\Sigma}^{A}$ and $n, k>0$. The quadruple

$$
\mathcal{A}_{n, k}=\left(A^{n}, \Sigma^{k},\left(\delta_{\mathbf{x}}: A^{n} \rightarrow A^{n}\right)_{\mathbf{x} \in \Sigma^{k}},\left(\rho_{\mathbf{u}}: \Sigma^{k} \rightarrow \Sigma^{k}\right)_{\mathbf{u} \in A^{n}}\right)
$$

is a Mealy automaton in $\mathfrak{T}_{A^{n}}^{\Sigma^{k}} \times \mathfrak{T}_{\Sigma^{k}}^{A^{n}}$ that we call the Mealy automaton of order $(n, k)$ associated with $\mathcal{A}$. Observe that $\mathcal{A}_{1,1}=\mathcal{A}$.

In Fig. 3. we show the Mealy automaton of order $(2,1)$ associated with the Mealy automaton of Fig. 2,

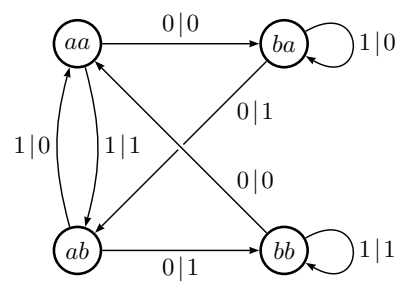

Figure 3: Mealy automaton of order $(2,1)$.

\subsection{Helix graph}

We have already seen two equivalent ways of presenting a Mealy automaton: $(i)$ as a quadruple $(A, \Sigma, \delta, \rho),(i i)$ as a labelled directed graph (see Fig. 2). We propose here a third and original one which turns out to be very convenient.

The helix graph $\mathcal{H}$ of a Mealy automaton $\mathcal{A}=(A, \Sigma, \delta, \rho)$ is the directed graph with nodes $A \times \Sigma$ and $\operatorname{arcs}(x, i) \longrightarrow\left(\delta_{i}(x), \rho_{x}(i)\right)$ for all $(x, i)$. The helix graph $\mathcal{H}_{n, k}$ of order $(n, k)$ associated with $\mathcal{A}$ is the helix graph of $\mathcal{A}_{n, k}$. In Fig. [4 we have represented the helix graph of the Mealy automaton of Fig. 2 .

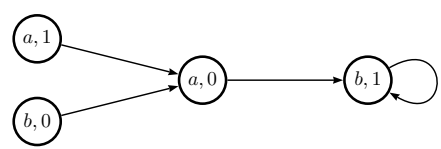

Figure 4: Helix graph.

Bireversible automata have a nice characterization using the helix graph. 
Lemma 2.2 Consider an IR-automaton $\mathcal{A}$ with helix graph $\mathcal{H}$. We have:

$$
[\mathcal{A} \text { bireversible }] \Longleftrightarrow[\mathcal{H} \text { union of cycles }] .
$$

Proof. Define the directed graph $\widetilde{\mathcal{H}}$ as follows:

- nodes: $A^{-1} \times \Sigma^{-1}$,

- $\operatorname{arcs}:\left(x^{-1}, i^{-1}\right) \longrightarrow\left(y^{-1}, j^{-1}\right)$ if $(y, j) \longrightarrow(x, i)$ is an arc of $\mathcal{H}$.

If $\operatorname{did} \mathfrak{i}(\mathcal{A})$ is a Mealy automaton, then $\widetilde{\mathcal{H}}$ is its helix graph.

Conversely, assume that $\mathcal{A}$ is an IR-automaton and that $\mathcal{H}$ is a union of cycles. Consider a node $(y, j)$ of $\mathcal{H}$ : it has a unique predecessor in $\mathcal{H}_{1,1}$.

\subsection{Automaton (semi)group}

Let $\mathcal{A}=(A, \Sigma, \delta, \rho)$ be a Mealy automaton. We view $\mathcal{A}$ as an automaton with an input and an output tape, thus defining mappings from input words over $\Sigma$ to output words over $\Sigma$. Formally, for $x \in A$, the map $\rho_{x}: \Sigma^{*} \rightarrow \Sigma^{*}$, extending $\rho_{x}: \Sigma \rightarrow \Sigma$, is defined by:

$$
\rho_{x}(\mathbf{u})=\mathbf{v} \quad \text { if } \quad \exists y, x \stackrel{\mathbf{u} \mid \mathbf{v}}{\longrightarrow} y .
$$

By convention, the image of the empty word is itself. The mapping $\rho_{x}$ is length-preserving and prefix-preserving (the prefix of the image is the image of the prefix). It satisfies

$$
\forall u \in \Sigma, \forall \mathbf{v} \in \Sigma^{*}, \quad \rho_{x}(u \mathbf{v})=\rho_{x}(u) \rho_{\delta_{u}(x)}(\mathbf{v}) .
$$

We can also use (3) to define $\rho_{x}: \Sigma^{*} \rightarrow \Sigma^{*}$ inductively starting from $\rho_{x}: \Sigma \rightarrow \Sigma$. We say that $\rho_{x}$ is the production function associated with $(\mathcal{A}, x)$. For $\mathbf{u}=u_{1} \cdots u_{n} \in A^{n}, n>0$, set $\rho_{\mathbf{u}}: \Sigma^{*} \rightarrow \Sigma^{*}, \rho_{\mathbf{u}}=\rho_{u_{n}} \circ \cdots \circ \rho_{u_{1}}$.

Definition 2.3 Consider $\mathcal{A} \in \mathfrak{T}_{A}^{\Sigma} \times \mathfrak{T}_{\Sigma}^{A}$. The semigroup of mappings from $\Sigma^{*}$ to $\Sigma^{*}$ generated by $\rho_{x}, x \in A$, is called the semigroup of $\mathcal{A}$ and is denoted by $\langle\mathcal{A}\rangle_{+}$. Assume that $\mathcal{A} \in \mathfrak{T}_{A}^{\Sigma} \times \mathfrak{S}_{\Sigma}^{A}$. The group of mappings from $\Sigma^{*}$ to $\Sigma^{*}$ generated as a group by $\rho_{x}, x \in A$, is called the group of $\mathcal{A}$ and is denoted by $\langle\mathcal{A}\rangle$.

The above definition makes sense. Indeed if $\mathcal{A} \in \mathfrak{T}_{A}^{\Sigma} \times \mathfrak{S}_{\Sigma}^{A}$, then the production mapping $\rho_{x}$ associated with $(\mathcal{A}, x)$ is a bijection from $\Sigma^{*}$ to $\Sigma^{*}$. The inverse bijection $\rho_{x}^{-1}: \Sigma^{*} \rightarrow \Sigma^{*}$ is the production mapping $\rho_{x^{-1}}$ associated with $\left(\mathcal{A}^{-1}, x^{-1}\right)$, where $\mathcal{A}^{-1}$ is the inverse Mealy automaton defined in (2). Therefore, we have

$$
\langle\mathcal{A}\rangle_{+}=\left\{\rho_{\mathbf{u}}, \mathbf{u} \in A^{*}\right\}, \quad\langle\mathcal{A}\rangle=\left\{\rho_{\mathbf{u}}, \mathbf{u} \in\left(A \sqcup A^{-1}\right)^{*}\right\} .
$$

Lemma 2.4 Let $\mathcal{A}$ be an IR-automaton. Then we have $\langle\mathcal{A}\rangle=\left\langle\mathcal{A}^{-1}\right\rangle=\left\langle\mathcal{A} \sqcup \mathcal{A}^{-1}\right\rangle=\left\langle\mathcal{A} \sqcup \mathcal{A}^{-1}\right\rangle_{+}$, where $\mathcal{A} \sqcup \mathcal{A}^{-1}$ is the Mealy automaton whose set of transitions is the union of the ones of $\mathcal{A}$ and $\mathcal{A}^{-1}$. Furthermore, if either $\langle\mathcal{A}\rangle$ or $\langle\mathcal{A}\rangle_{+}$is finite, then we have $\langle\mathcal{A}\rangle=\langle\mathcal{A}\rangle_{+}$.

Proof. The first statement follows directly from the definitions. Suppose that $\langle\mathcal{A}\rangle_{+}$is finite and let $x$ be one of its elements. Since the semigroup $\langle\mathcal{A}\rangle_{+}$is finite, there exist $k$ and $n$ such that $x^{n+k}=x^{k}$. So we have $x^{n}=1$ in the group $\langle\mathcal{A}\rangle$. Hence the inverse of $x$ is $x^{n-1}$ which belongs to the semigroup $\langle\mathcal{A}\rangle_{+}$. So we have $\langle\mathcal{A}\rangle=\langle\mathcal{A}\rangle_{+}$. Assume now that $\langle\mathcal{A}\rangle$ is finite. Since the semigroup $\langle\mathcal{A}\rangle_{+}$naturally embeds into the group $\langle\mathcal{A}\rangle$, it is also finite.

Definition 2.5 A semigroup $M$ is called an automaton semigroup if there exists a Mealy automaton $\mathcal{A}$ such that $M=\langle\mathcal{A}\rangle_{+}$. A group $G$ is called an automaton group if there exists an invertible Mealy automaton $\mathcal{A}$ such that $G=\langle\mathcal{A}\rangle$. In both cases, we say that $\mathcal{A}$ generates the (semi)group. 
Denote dually by $\delta_{i}: A^{*} \rightarrow A^{*}, i \in \Sigma$, the production mappings associated with the dual Mealy automaton $\mathfrak{d}(\mathcal{A})$. For $\mathbf{v}=v_{1} \cdots v_{n} \in \Sigma^{n}, n>0$, set $\delta_{\mathbf{v}}: A^{*} \rightarrow A^{*}, \delta_{\mathbf{v}}=\delta_{v_{n}} \circ \cdots \circ \delta_{v_{1}}$.

A pair of Mealy automata $\{\mathcal{A}, \mathfrak{d}(\mathcal{A})\}$ generates a pair of (semi)groups.

Examples of automata (semi)groups are given in Table 2,

The two following propositions complement each other. Proposition 2.7 is proved by Nekrashevych for a pair of dual bireversible Mealy automata [24, Lem.1.10.6]. For the sake of completeness, we provide a similar proof in the general case.

Proposition 2.6 Let $G$ and $H$ be two finite semigroups. There exists a Mealy automaton $\mathcal{A}$ such that $\langle\mathcal{A}\rangle_{+}=G$ and $\langle\mathfrak{d}(\mathcal{A})\rangle_{+}=H$. Let $G$ and $H$ be two finite groups. There exists an IR-automaton $\mathcal{A}$ such that $\langle\mathcal{A}\rangle=G$ and $\langle\mathfrak{d}(\mathcal{A})\rangle=H$.

Proof. We carry out the proof for groups. The argument is similar for semigroups.

Any finite group is a subgroup of a permutation group. Let $\Sigma_{1}$ and $A_{2}$ be two finite sets such that $G$ is a subgroup of $\mathfrak{S}_{\Sigma_{1}}$ and $H$ is a subgroup of $\mathfrak{S}_{A_{2}}$. Let $A_{1} \subset \mathfrak{S}_{\Sigma_{1}}$ be a set of generators of $G$, let $\Sigma_{2} \subset \mathfrak{S}_{A_{2}}$ be a set of generators of $H$.

Set $A=A_{1} \times A_{2}$ and $\Sigma=\Sigma_{1} \times \Sigma_{2}$. Consider the Mealy automaton $\mathcal{A}$ with states $A$, alphabet $\Sigma$, and transitions

$$
(a, b) \stackrel{(i, j) \mid(a(i), j)}{\longrightarrow}(a, j(b)) .
$$

Denote the corresponding mappings by $\delta$ and $\rho$. Clearly, for $(a, b) \in A_{1} \times A_{2}$ and $\left(a, b^{\prime}\right) \in$ $A_{1} \times A_{2}$, we have $\rho_{(a, b)}=\rho_{\left(a, b^{\prime}\right)}$ and we denote this mapping by $\rho_{a}: \Sigma^{*} \rightarrow \Sigma^{*}$. We have, $\forall a \in A_{1}, \forall\left(i_{1}, j_{1}\right) \cdots\left(i_{n}, j_{n}\right) \in \Sigma^{*}$,

$$
\rho_{a}\left(\left(i_{1}, j_{1}\right) \cdots\left(i_{n}, j_{n}\right)\right)=\left(a\left(i_{1}\right), j_{1}\right)\left(a\left(i_{2}\right), j_{2}\right) \cdots\left(a\left(i_{n}\right), j_{n}\right) .
$$

So the group generated by $\left(\rho_{a}: \Sigma^{*} \rightarrow \Sigma^{*}\right)_{a \in A_{1}}$ is isomorphic to the group generated by $\left(a: \Sigma_{1} \rightarrow\right.$ $\left.\Sigma_{1}\right)_{a \in A_{1}}$. That is $\langle\mathcal{A}\rangle=G$. Similarly, $\langle\mathfrak{d}(\mathcal{A})\rangle=H$.

Proposition 2.7 Let $\mathcal{A}$ be a Mealy automaton. The semigroup $\langle\mathcal{A}\rangle_{+}$is finite if and only if the semigroup $\langle\mathfrak{d}(\mathcal{A})\rangle_{+}$is finite.

Proposition 2.7 extends to groups using Lemma 2.4

Proof. Set $\mathcal{A}=(A, \Sigma, \delta, \rho)$ and assume that $\langle\mathfrak{d}(\mathcal{A})\rangle_{+}=\left\{\delta_{\mathbf{u}}: A^{*} \rightarrow A^{*}, \mathbf{u} \in \Sigma^{*}\right\}$ is finite. Consider the Cayley graph $\mathcal{G}$ of $\langle\mathfrak{d}(\mathcal{A})\rangle_{+}$with respect to the set of generators $\Sigma$, see the left of the figure just below. Now fix $\mathbf{w} \in A^{*}$ and recall that

$$
\rho_{\mathbf{w}}\left(u_{1} u_{2} \cdots u_{n}\right):=\rho_{\mathbf{w}}\left(u_{1}\right) \rho_{\delta_{u_{1}}(\mathbf{w})}\left(u_{2}\right) \rho_{\delta_{u_{1} u_{2}}(\mathbf{w})}\left(u_{3}\right) \cdots \rho_{\delta_{u_{1} u_{2} \cdots u_{n-1}}(\mathbf{w})}\left(u_{n}\right),
$$

for all $u_{1} u_{2} \cdots u_{n} \in \Sigma^{*}$. This shows that $\rho_{\mathbf{w}}$ can also be described as the output map of a letter-to-letter transducer built upon $\mathcal{G}$, see the right of the figure.

$$
\delta_{\mathrm{u}} \stackrel{i}{\longrightarrow} \delta_{\mathrm{u} i \mathrm{u}} \stackrel{i \mid \rho_{\delta_{\mathrm{u}}(\mathbf{w})}(i)}{\longrightarrow} \delta_{\mathrm{u} i}
$$

Now observe that there is only a finite number of possible different transducers built on $\mathcal{G}$, which is equal to the number of different mappings from $\langle\mathfrak{d}(\mathcal{A})\rangle_{+}$to $\mathfrak{T}_{\Sigma}$. We conclude that $\#\langle\mathcal{A}\rangle_{+} \leq$ $(\# \Sigma)^{(\# \Sigma)\left(\#\langle\mathfrak{o}(\mathcal{A})\rangle_{+}\right)}$.

The growth of a Mealy automaton is defined as the growth of the number of different elements $\rho_{\mathbf{u}}, \mathbf{u} \in A^{n}$, as a function of $n$, see [7, 15]. Automata generating finite (semi)groups are those of finite growth. Looking at the 2-letter 2-state automata, it appears that it is the only growth class within the known growth classes (finite, polynomial, intermediate and exponential) to be stable by dualization.

Let $\mathcal{A}$ be an IR-automaton. Recall that $\langle\mathcal{A}\rangle=\left\langle\mathcal{A} \sqcup \mathcal{A}^{-1}\right\rangle$. In words, considering the states and their inverses does not modify the generated group. We can also consider the letters and their inverses. Set $\widetilde{\mathcal{A}}=\mathcal{A}^{\prime} \sqcup\left(\mathcal{A}^{\prime}\right)^{-1}$ where $\mathcal{A}^{\prime}=\mathfrak{d}\left(\mathfrak{d}(\mathcal{A}) \sqcup \mathfrak{d}(\mathcal{A})^{-1}\right)$. The Mealy automaton $\widetilde{\mathcal{A}}$ is the extension of $\mathcal{A}$ with stateset $A \sqcup A^{-1}$ and alphabet $\Sigma \sqcup \Sigma^{-1}$.

Next result is a corollary of Proposition 2.7 and Lemma 2.4 
Table 2: Examples of automata (semi)groups.

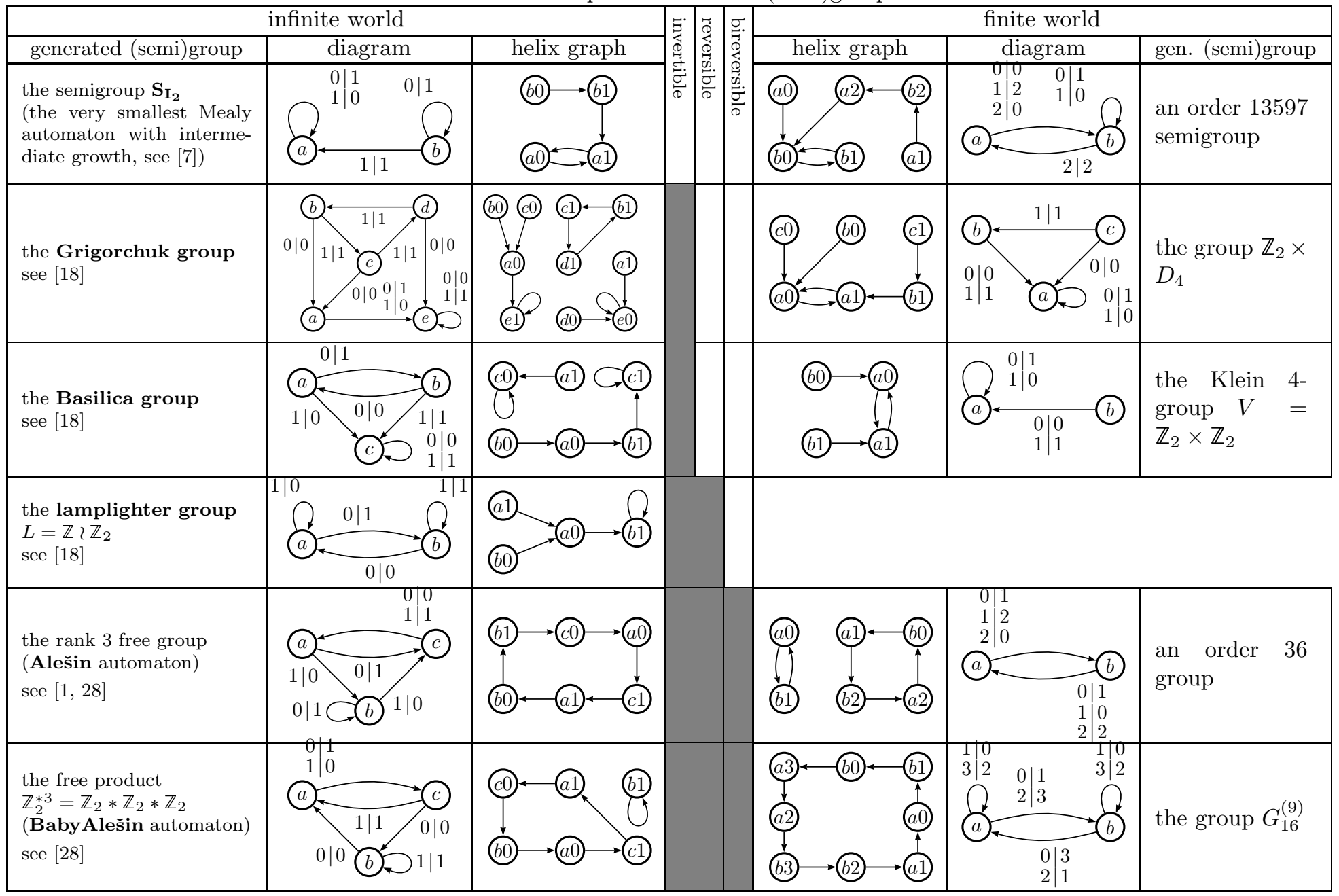


Corollary 2.8 Let $\mathcal{A}$ be an IR-automaton. The groups $\langle\mathcal{A}\rangle$ and $\langle\tilde{\mathcal{A}}\rangle$ are either both finite or both infinite.

The above groups are not necessary equal. Consider for instance the automaton $\mathcal{A}$ generating $G_{16}^{(9)}$ in Table 2, we have $|\langle\mathcal{A}\rangle|=16$ and $|\langle\widetilde{\mathcal{A}}\rangle|=64$.

\section{Reduction of Mealy automata and finiteness}

Here we define the $\mathfrak{m} \mathfrak{d}$-reduction of Mealy automata which provides a sufficient condition of finiteness. The condition is not necessary and two counterexamples are provided.

\subsection{Minimization of a Mealy automaton}

Definition 3.1 Let $\mathcal{A}=(A, \Sigma, \delta, \rho)$ be a Mealy automaton. An equivalence $\equiv$ on $A$ is a congruence for $\mathcal{A}$ if

$$
[\forall x, y \in A, x \equiv y] \Longrightarrow\left[\forall i \in \Sigma, \rho_{x}(i)=\rho_{y}(i) \text { and } \delta_{i}(x) \equiv \delta_{i}(y)\right]
$$

The Nerode equivalence on $A$ is the coarsest congruence for $\mathcal{A}$.

The Nerode equivalence is the limit of the sequence $\left(\equiv_{k}\right)$ of increasingly finer equivalences defined recursively by:

$$
\begin{aligned}
\forall x, y \in A, \quad x \equiv_{0} y & \Longleftrightarrow \forall i \in \Sigma, \rho_{x}(i)=\rho_{y}(i), \\
\forall k \geqslant 0, x \equiv_{k+1} y & \Longleftrightarrow x \equiv_{k} y \text { and } \forall i \in \Sigma, \delta_{i}(x) \equiv_{k} \delta_{i}(y) .
\end{aligned}
$$

Since the set $A$ is finite, this sequence is ultimately constant; moreover if two consecutive equivalences are equal, the sequence remains constant from this point. The limit is therefore computable. For every $x$ in $A$, we denote by $[x]$ the class of $x$ w.r.t. the Nerode equivalence.

Definition 3.2 Let $\mathcal{A}=(A, \Sigma, \delta, \rho)$ be a Mealy automaton and let $\equiv$ be the Nerode equivalence on $\mathcal{A}$. The minimization of $\mathcal{A}$ is the Mealy automaton $\mathcal{A} / \equiv=(A / \equiv, \Sigma, \tilde{\delta}, \tilde{\rho})$, where for every $(x, i)$ in $A \times \Sigma, \tilde{\delta}_{i}([x])=\left[\delta_{i}(x)\right]$ and $\tilde{\rho}_{[x]}(i)=\rho_{x}(i)$.

This definition is consistent with the minimization of "deterministic finite automata", where instead of considering the production functions $\left(\rho_{x}\right)_{x}$, the computation of the congruence is initiated by the separation between terminal and non-terminal states.

Lemma 3.3 Let $\mathcal{A}=(A, \Sigma, \delta, \rho)$ be a Mealy automaton, and let $\mathcal{A} / \equiv$ be its minimization. The function on $\Sigma^{*}$ generated by $x$ in $\mathcal{A}$ is equal to the function generated by $[x]$ in $\mathcal{A} / \equiv$. Therefore, the Mealy automata $\mathcal{A}$ and $\mathcal{A} / \equiv$ generate the same semigroup.

Proof. Let $\left(\mathbf{i}_{n}\right)_{n \in \mathbb{N}}$ be a sequence of words of $\Sigma^{*}$ such that for all integer $n$, the length of $\mathbf{i}_{n}$ is $n$ and $\mathbf{i}_{n}$ is a prefix of $\mathbf{i}_{n+1}: \mathbf{i}_{n+1}=\mathbf{i}_{n} i_{n+1}$, where $i_{n+1} \in \Sigma$. We prove by induction on $n$ that for every $x$ of $A$, we have $\rho_{x}=\tilde{\rho}_{[x]}$ on $\Sigma^{n}$. It is obviously true for $n=0$. If $n>0$ :

$$
\begin{aligned}
\rho_{x}\left(\mathbf{i}_{n}\right) & =\rho_{x}\left(\mathbf{i}_{n-1}\right) \rho_{\delta_{\mathbf{i}_{n-1}}(x)}\left(i_{n}\right) \\
& =\tilde{\rho}_{[x]}\left(\mathbf{i}_{n-1}\right) \tilde{\rho}_{\left[\delta_{\mathbf{i}_{n-1}}(x)\right]}\left(i_{n}\right) \\
& =\tilde{\rho}_{[x]}\left(\mathbf{i}_{n-1}\right) \tilde{\rho}_{\tilde{\delta}_{\mathbf{i}_{n-1}}([x])}\left(i_{n}\right)=\tilde{\rho}_{[x]}\left(\mathbf{i}_{n}\right) .
\end{aligned}
$$




\subsection{The mo-reduction of Mealy automata}

Observe that the minimization of a Mealy automaton with a minimal dual can make the dual automaton non-minimal.

Definition 3.4 A pair of dual Mealy automata is reduced if both Mealy automata are minimal. Let $\mathfrak{m}$ be the operation of minimization; recall that $\mathfrak{d}$ is the operation of dualization. The $\mathfrak{m} \mathfrak{d}$ reduction of a Mealy automaton consists in minimizing the automaton or its dual until the resulting pair of dual Mealy automata is reduced.

If both a Mealy automaton and its dual automaton are non-minimal, the procedure of moreduction seems to be dependent on the first automaton chosen for the minimization. The reduction is actually confluent:

Proposition 3.5 If $(\mathcal{A}, \mathcal{B})$ is a pair of dual Mealy automata, the reduced pair obtained by minimizing $\mathcal{A}$ first is the same as the one obtained by minimizing $\mathcal{B}$ first.

Proof. If $(\mathcal{A}, \mathcal{B})$ is reduced, both Mealy automata are minimal, and the proposition trivially holds.

Otherwise, the proof is by induction on the total number of states in $\mathcal{A}$ and $\mathcal{B}$. Let $\left(\mathcal{A}_{1}, \mathcal{B}_{1}\right)$ be the pair obtained by minimizing $\mathcal{A}$ and let $\left(\mathcal{A}_{2}, \mathcal{B}_{2}\right)$ be the pair obtained by minimizing $\mathcal{B}$. Let us set $\mathcal{A}=(A, \Sigma, \delta, \rho), \mathcal{A}_{1}=\left(A_{1}, \Sigma, \delta^{(1)}, \rho^{(1)}\right)$, and $\mathcal{A}_{2}=\left(A, \Sigma_{2}, \delta^{(2)}, \rho^{(2)}\right)$. Let $\equiv_{1}$ and $\equiv_{2}$ be the congruences on $\mathcal{A}$ and $\mathcal{B}$ such that $A_{1}=A / \equiv_{1}$ and $\Sigma_{2}=\Sigma / \equiv_{2}$. We show that $\equiv_{1}$ is a congruence on $\mathcal{A}_{2}$. Let $x$ and $y$ be in $A$ such that $x \equiv_{1} y$. Then, for every $i$ in $\Sigma, \rho_{x}(i)=\rho_{y}(i)$ and therefore, $\rho_{x}^{(2)}([i])=\left[\rho_{x}(i)\right]=\left[\rho_{y}(i)\right]=\rho_{y}^{(2)}([i])$; besides, $\delta_{[i]}^{(2)}(x)=\delta_{i}(x) \equiv_{1} \delta_{i}(y)=\delta_{[i]}^{(2)}(y)$. Hence, $\equiv_{1}$ is a congruence on $\mathcal{A}_{2}$ and, likewise, $\equiv_{2}$ is a congruence on $\mathcal{B}_{1}$. We consider now the Mealy automaton $\mathcal{A}^{\prime}=\left(A_{1}, \Sigma_{2}, \delta^{\prime}, \rho^{\prime}\right)$ which is the quotient of $\mathcal{A}_{2}$ with respect to $\equiv_{1}$, and $\mathcal{B}^{\prime}=\left(\Sigma_{2}, A_{1}, \rho^{\prime \prime}, \delta^{\prime \prime}\right)$ which is the quotient of $\mathcal{B}_{1}$ w.r.t. $\equiv_{2}$. For every $x$ in $A$ and every $i$ in $\Sigma$, it holds:

$$
\delta^{\prime \prime}{ }_{[i]}([x])=\delta_{i}^{(1)}([x])=\left[\delta_{i}(x)\right]=\left[\delta_{[i]}^{(2)}(x)\right]=\delta_{[i]}^{\prime}([x]) .
$$

Thus, $\delta^{\prime \prime}=\delta^{\prime}$ and likewise $\rho "=\rho^{\prime}$.

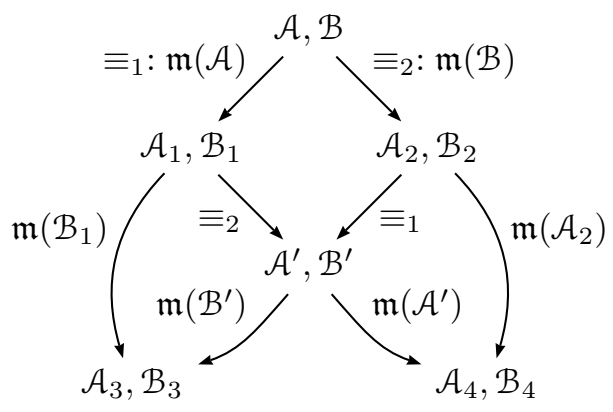

Consider now $\mathcal{A}_{2}=\left(A, \Sigma_{2}, \delta^{(2)}, \rho^{(2)}\right)$ and $\mathcal{A}^{\prime}=\left(A_{1}, \Sigma_{2}, \delta^{\prime}, \rho^{\prime}\right)$. Clearly, applying the coarsest congruences respectively on $A$ in $\mathcal{A}_{2}$ and $A_{1}$ in $\mathcal{A}^{\prime}$ will result in the same minimized Mealy automaton $\mathcal{A}_{4}$. The minimized Mealy automaton $\mathcal{B}_{3}$ is defined similarly starting from either $\mathcal{B}_{1}$ or $\mathcal{B}^{\prime}$. Let $\mathcal{B}_{4}$ be the dual of $\mathcal{A}_{4}$, and let $\mathcal{A}_{3}$ be the dual of $\mathcal{B}_{3}$. By construction, the pair $\left(\mathcal{A}_{3}, \mathcal{B}_{3}\right)$ (resp. $\left.\left(\mathcal{A}_{4}, \mathcal{B}_{4}\right)\right)$ is the one obtained from $(\mathcal{A}, \mathcal{B})$ by minimizing first $\mathcal{A}$ (resp. $\mathcal{B}$ ) then $\mathcal{B}$ (resp. $\mathcal{A})$. But the pair $\left(\mathcal{A}_{3}, \mathcal{B}_{3}\right)$ (resp. $\left.\left(\mathcal{A}_{4}, \mathcal{B}_{4}\right)\right)$ is also the one obtained by applying one minimization step starting from $\left(\mathcal{A}^{\prime}, \mathcal{B}^{\prime}\right)$. Observe that the pair $\left(\mathcal{A}^{\prime}, \mathcal{B}^{\prime}\right)$ has a number of states strictly smaller than the one of $(\mathcal{A}, \mathcal{B})$. By induction hypothesis, starting from $\left(\mathcal{A}^{\prime}, \mathcal{B}^{\prime}\right)$, the $\mathfrak{m} \mathfrak{d}$-reduction does not depend on the first minimization step, which proves the result. 


\subsection{A sufficient condition for finiteness}

A trivial Mealy automaton is a Mealy automaton with one state over a one-letter alphabet. It clearly generates the trivial group.

Theorem 3.6 If the $\mathfrak{m} \mathfrak{d}$-reduction of a Mealy automaton (resp. an invertible Mealy automaton) leads to a trivial Mealy automaton, then the automaton generates a finite semigroup (resp. a finite group).

Proof. Let $(\mathcal{A}, \mathcal{B})$ be a pair of dual Mealy automata and assume that there exists a sequence of dual Mealy automata $\left(\left(\mathcal{A}_{k}, \mathcal{B}_{k}\right)\right)_{k \in[0, m]}$ such that $\left(\mathcal{A}_{0}, \mathcal{B}_{0}\right)=(\mathcal{A}, \mathcal{B}),\left(\mathcal{A}_{m}, \mathcal{B}_{m}\right)$ is trivial and, for every $k \in[1, m]$, either $\mathcal{A}_{k}$ is the minimization of $\mathcal{A}_{k-1}$ or $\mathcal{B}_{k}$ is the minimization of $\mathcal{B}_{k-1}$.

By Proposition 2.7. for every $k$, if $\mathcal{A}_{k}$ or $\mathcal{B}_{k}$ generates a finite semigroup, both automata do. Obviously, $\mathcal{A}_{m}$ and $\mathcal{B}_{m}$ both generate the trivial group. We prove that if $\mathcal{A}_{k}$ generates a finite semigroup, so does $\mathcal{A}_{k-1}$. If $\mathcal{A}_{k}$ is the minimization of $\mathcal{A}_{k-1}$, by Lemma 3.3, they both generate the same semigroup. Otherwise, $\mathcal{B}_{k}$ is the minimization of $\mathcal{B}_{k-1}$. Then $\mathcal{B}_{k}$ generates a finite semigroup (Prop. 2.7), so does $\mathcal{B}_{k-1}$ (Lem. 3.3), and thus $\mathcal{A}_{k-1}$ (Prop. 2.7). Therefore $\mathcal{A}$ generates a finite semigroup.

Let $\mathcal{A}$ be the following automaton:

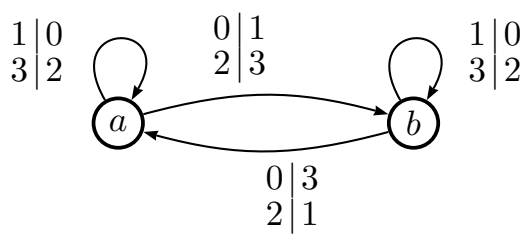

Let us compute the $\mathfrak{m} \mathfrak{d}$-reduced automaton of $\mathcal{A}$. 

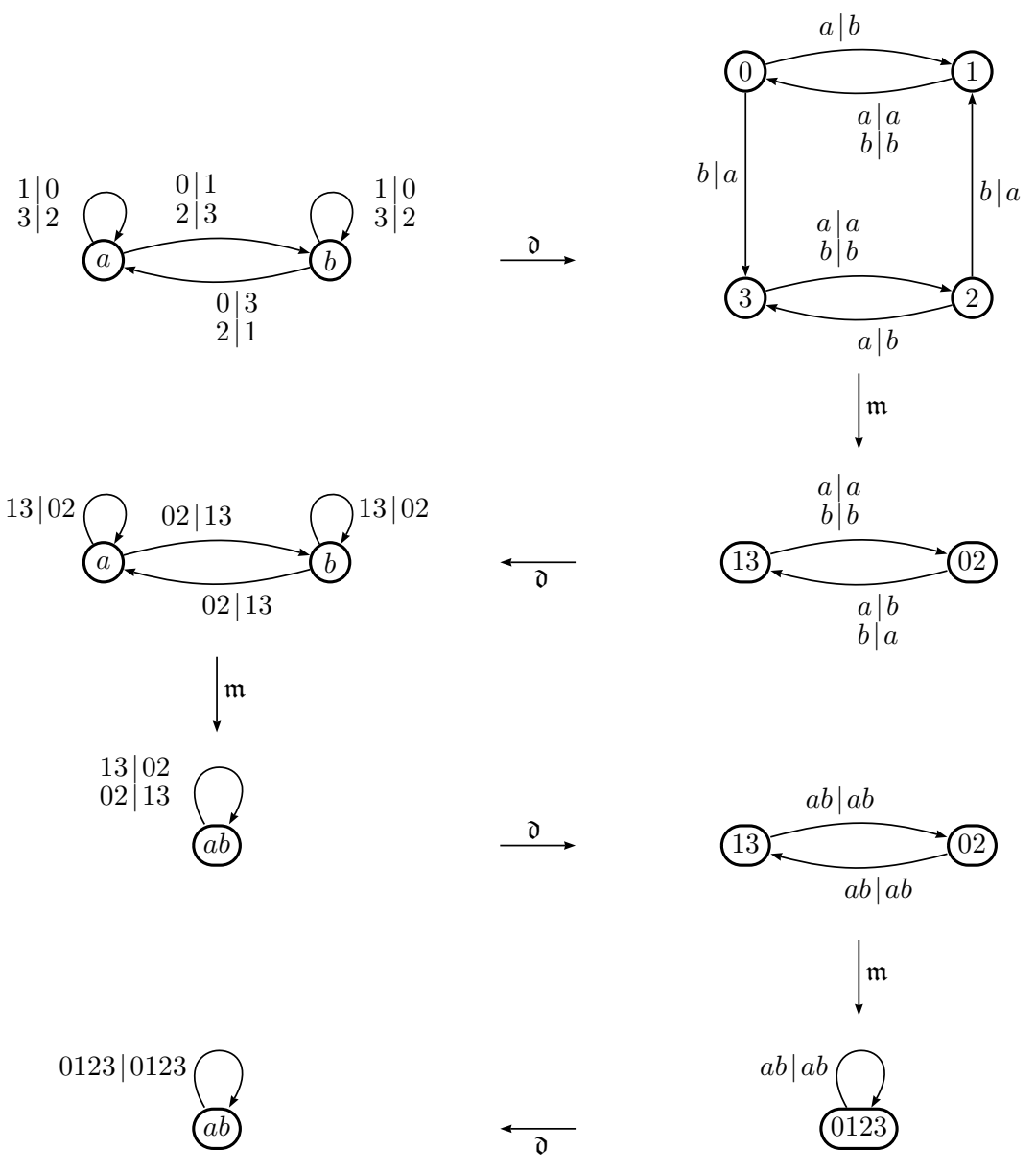

The group generated by $\mathcal{A}$ is finite and can be shown to be isomorphic to $G_{16}^{(9)}$, that is, the group of order 16 with presentation

$$
\left\langle a, b: a^{4}=b^{4}=a b a b=1, a b^{3}=b a^{3}\right\rangle .
$$

Now consider the family $\left(\mathcal{M N}_{p, q}^{\sharp}\right)$ of bireversible $p$-letter $q$-state Mealy automata:

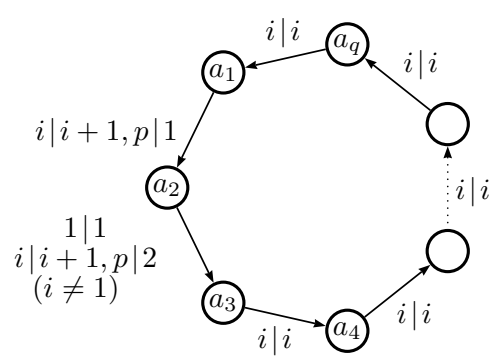

One can check that $(\mathfrak{d m o m})\left(\mathcal{M}_{p, q}^{\sharp}\right)$ is trivial for any $p$ and $q$. Hence by Theorem 3.6, the groups $\left\langle\mathcal{N}_{p, q}^{\sharp}\right\rangle$ are all finite. In fact and independently, the group $\left\langle\mathcal{N}_{p, q}^{\sharp}\right\rangle$ can be identified with $\mathfrak{S}_{q}^{p}$. For comparison, the packages $\mathbf{F R}$ and automgrp both fail to decide finiteness of $\left\langle\mathcal{M} \mathcal{M}_{p, q}^{\sharp}\right\rangle$ (except for very small values of $p, q$ ).

\subsection{This sufficient condition is not necessary}

The following Mealy automaton is $\mathfrak{m} \mathfrak{d}$-reduced, but it generates a finite semigroup of order 6 : it provides a counterexample to the converse of Theorem 3.6. 


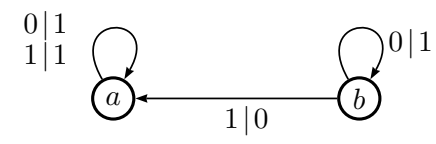

There also exist counterexamples among bireversible Mealy automata. Consider the order 8 dihedral group viewed as generated by a reflection $\sigma$ and by a product $\mu=\rho \sigma$ with a rotation:

$$
D_{4}=\left\langle\sigma, \mu: \sigma^{2}=\mu^{2}=(\sigma \mu)^{4}=1\right\rangle .
$$

It is generated by the bireversible Mealy automaton of Fig. 5. This ad-hoc automaton is its own dual and is $\mathfrak{m} \mathfrak{d}$-reduced.

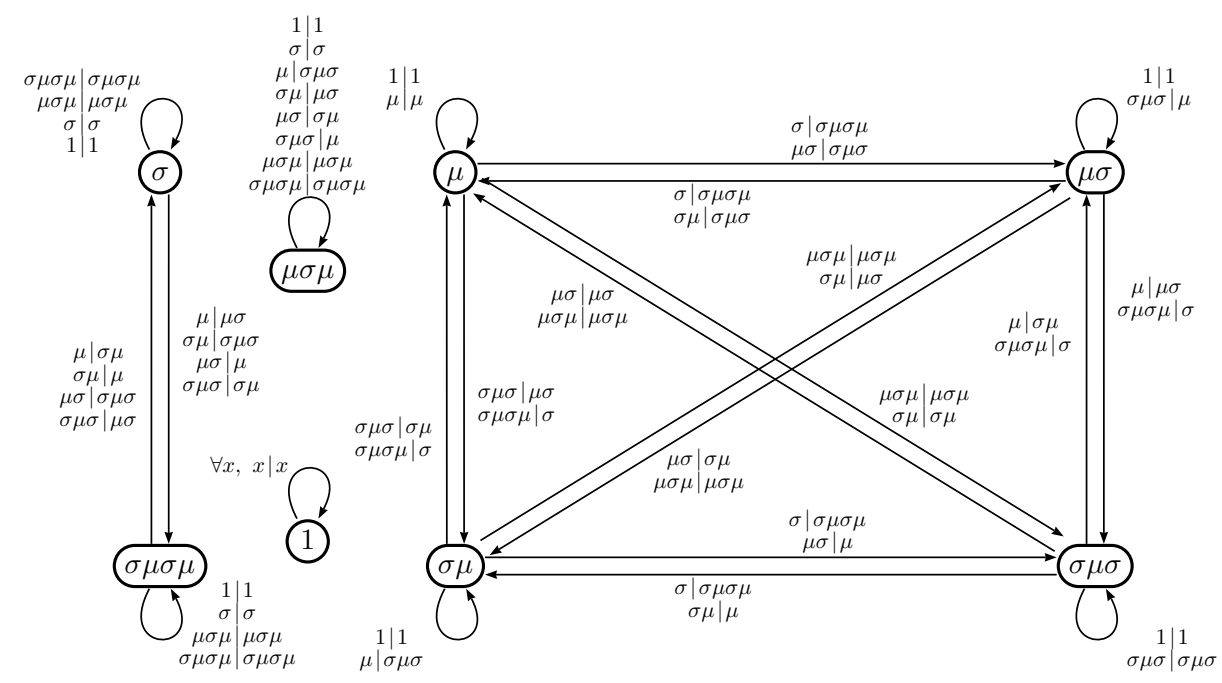

Figure 5: An $\mathfrak{m} \mathfrak{d}$-reduced non-trivial IR-automaton whose group is finite.

\section{Helix graphs and finiteness}

In this section, we concentrate on IR-automata and show the pertinence of helix graphs for the finiteness problem.

\subsection{A necessary condition for finiteness}

To prove the results in this section, it is convenient to use a graphical representation in which $A$ and $\Sigma$ play symmetrical roles. Consider $(x, i) \in A \times \Sigma$ with $\delta_{i}(x)=y$ and $\rho_{x}(i)=j$. The corresponding transition $x \stackrel{i \mid j}{\longrightarrow} y$ is represented by the cross-transition:

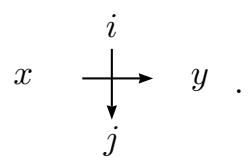

The automaton $\mathcal{A}$ is identified with the set of its cross-transitions (of cardinality $|A| \times|\Sigma|$ ).

A path in $\mathcal{A}($ resp. in $\mathfrak{d}(\mathcal{A})$ ) is represented by an horizontal (resp. vertical) cross-diagram obtained by concatenating the crosses. We may also consider rectangular cross-diagrams of dimension 
$m \times n$, on which one can read the production functions of $\mathcal{A}_{m, n}$ and $\mathfrak{d}\left(\mathcal{A}_{m, n}\right)$. For instance the cross-

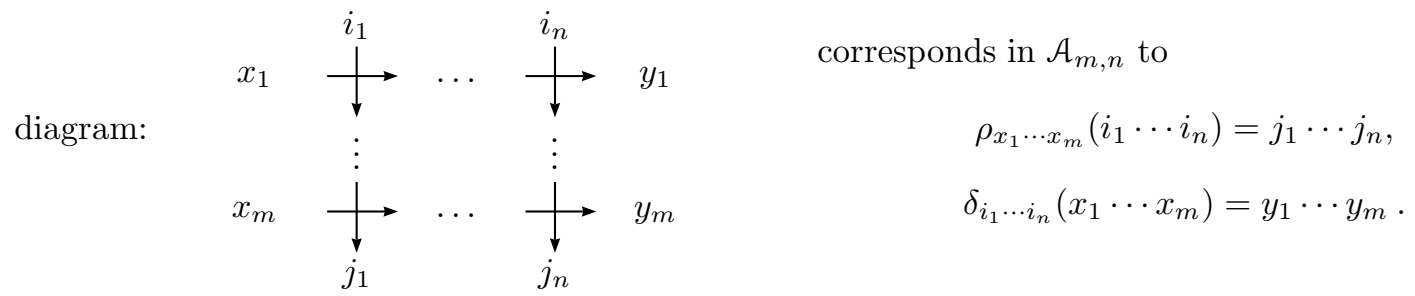

Replacing every cross by a square, we get the "square-diagrams" of [14.

Proposition 4.1 Let $\mathcal{A}$ be an IR-automaton. If the helix graph of order $(1,1)$ of $\mathcal{A}$ is a union of cycles, so are all the helix graphs (of any order) of $\mathcal{A}$.

Proof. Observe that a helix graph is a union of cycles if and only if any node has a predecessor. By assumption, $\mathcal{H}$ is a union of cycles, therefore, any $(x, u) \in A \times \Sigma$ has a predecessor. Now consider $(\mathbf{x}, \mathbf{u}) \in A^{m} \times \Sigma^{n}$ with $\mathbf{x}=x_{1} \cdots x_{m}$ and $\mathbf{u}=u_{1} \cdots u_{n}$. Let $\left(\tilde{x}_{m}, \tilde{u}_{n}\right)$ be the predecessor of $\left(x_{m}, u_{n}\right)$ in $\mathcal{H}$. Start with the cross of $\left(\tilde{x}_{m}, \tilde{u}_{n}\right)$ and $\left(x_{m}, u_{n}\right)$ (left of (4) ), and expand it step-by-step using the existence of predecessors in $\mathcal{H}$ (right of (4) for the first few steps).

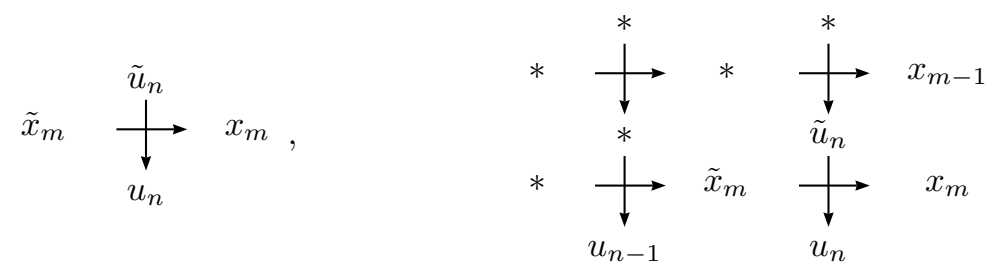

In the end we get a cross-diagram of dimension $m \times n$. The words on the west and north of the cross-diagram form a predecessor for $(\mathbf{x}, \mathbf{u})$.

Theorem 4.2 Let $\mathcal{A}$ be an IR-automaton. If $\langle\mathcal{A}\rangle$ is finite, then the helix graphs of $\mathcal{A}$ are unions of cycles.

Proof. By Proposition 4.1, it is sufficient to prove the result for order $(1,1)$. Consider $x \in A$ and $i \in \Sigma$. According to Proposition 2.7 $\langle\mathfrak{d}(\mathcal{A})\rangle$ is finite. Therefore, there exist $m, n>0$ such that $\rho_{x}^{m}=\rho_{x^{m}}=\operatorname{id}_{\langle\mathcal{A}\rangle}$ and $\delta_{i}^{n}=\delta_{i^{n}}=\operatorname{id}_{\langle\mathfrak{o}(\mathcal{A})\rangle}$. It implies that $x^{m} \stackrel{i^{n} \mid i^{n}}{\longrightarrow} x^{m}$ is a transition in the Mealy automaton of order $(m, n)$. The corresponding cross-diagram is represented below:

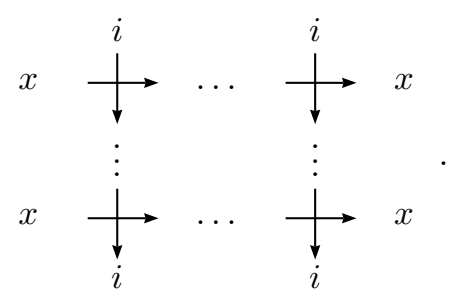

The south-east cross of the diagram provides a predecessor for $(x, i)$.

There exist IR-automata generating infinite groups whose helix graphs are union of cycles. The smallest examples are Alešin automata (see Table 21).

Next result follows directly from Lemma 2.2 and Theorem 4.2 .

Corollary 4.3 Consider an IR-automaton which is not bireversible. Then the group generated by the automaton is infinite. 


\subsection{A necessary and sufficient condition for finiteness}

The condition in next theorem is not effective. Hence, it does not directly lead to a decision procedure of finiteness.

Recall the construction and notation defined at the end of section 2.3 for an IR-automaton $\mathcal{A}$ with stateset $A$ and alphabet $\Sigma$, we denote by $\widetilde{A}$ the extension with stateset $A \sqcup A^{-1}$ and alphabet $\Sigma \sqcup \Sigma^{-1}$.

Theorem 4.4 Consider an IR-automaton $\mathcal{A}$. The group $\langle\mathcal{A}\rangle$ is finite if and only if there exists $K$ such that, for all $k, l$, the helix graphs $\mathcal{H}(k, l)$ of $\widetilde{\mathcal{A}}$ are unions of cycles of lengths bounded by $K$.

Proof. Assume first that $\langle\mathcal{A}\rangle$ is finite: so is $\langle\widetilde{\mathcal{A}}\rangle$ by Corollary 2.8. Theorem 4.2 shows that helix graphs of any order are unions of cycles. It remains to prove that the lengths of these cycles are uniformly bounded. By Proposition 2.7 the group $\langle\mathfrak{d}(\widetilde{\mathcal{A}})\rangle$ is finite as well. Let $\mathcal{C}$ be a cycle in a helix graph of $\widetilde{\mathcal{A}}$ and let $(\mathbf{u}, \mathbf{v}) \in\left(A \sqcup A^{-1}\right)^{*} \times\left(\Sigma \sqcup \Sigma^{-1}\right)^{*}$ be a node of this cycle. Each node of $\mathcal{C}$ is of the form $(h(\mathbf{u}), g(\mathbf{v}))$, where $g($ resp. $h)$ is an element of $\langle\widetilde{\mathcal{A}}\rangle(\operatorname{resp} .\langle\mathcal{d}(\widetilde{\mathcal{A}})\rangle)$. Since the nodes are pairwise distinct, the length of the cycle $\mathcal{C}$ is at most $\#\langle\widetilde{\mathcal{A}}\rangle \times \#\langle\mathfrak{d}(\widetilde{\mathcal{A}})\rangle$.

Let us prove the converse and assume that the group $\langle\mathcal{A}\rangle$ is infinite: so is $\langle\widetilde{\mathcal{A}}\rangle$ by Corollary 2.8 First we argue that the orders of the elements of $\langle\widetilde{\mathcal{A}}\rangle$ are unbounded. Indeed, automata groups are residually finite by construction since they act faithfully on rooted locally finite trees. Moreover it follows from Zelmanov's solution of the restricted Burnside problem [31, 32, 30] that any residually finite group with bounded torsion is finite. Since $\langle\widetilde{\mathcal{A}}\rangle$ is infinite, the orders of its elements are unbounded.

There exists either $\mathbf{x} \in\left(A \sqcup A^{-1}\right)^{*}$ such that the order of $\rho_{\mathbf{x}}$ is infinite, or a sequence $\left(\mathbf{x}_{n}\right)_{n \in \mathbb{N}} \subseteq$ $\left(A \sqcup A^{-1}\right)^{*}$ such that the sequence $\left(k_{n}\right)_{n}$ of orders of the elements $\rho_{\mathbf{x}_{n}}$ converges to infinity. We carry out the proof in the second case, the first one can be treated similarly. Let us concentrate on $\rho_{\mathbf{x}_{n}}$, element of order $k_{n}$ of $\langle\widetilde{\mathcal{A}}\rangle$. For all $1 \leq k<k_{n}$, there exists a word $\mathbf{u}_{k} \in\left(\Sigma \sqcup \Sigma^{-1}\right)^{*}$ such that $\rho_{\mathbf{x}_{n}}^{k}\left(\mathbf{u}_{k}\right)=\widetilde{\mathbf{u}}_{k} \neq \mathbf{u}_{k}$.

Say that a word $\mathbf{v} \in\left(\Sigma \sqcup \Sigma^{-1}\right)^{*}$ is unitary if $\delta_{\mathbf{v}}$ is the identity of $\langle\mathfrak{d}(\widetilde{\mathcal{A}})\rangle$. Since $\langle\mathfrak{d}(\widetilde{\mathcal{A}})\rangle$ is a group, the word $\mathbf{u}_{k}$ can be extended into a unitary word $\mathbf{u}_{k} \mathbf{v}_{k}$. Set $\mathbf{w}_{n}=\mathbf{u}_{1} \mathbf{v}_{1} \cdots \mathbf{u}_{k_{n}-1} \mathbf{v}_{k_{n}-1}$. By construction, we have: $\rho_{\mathbf{x}_{n}}\left(\mathbf{w}_{n}\right)=\widetilde{\mathbf{u}}_{1} \cdots \neq \mathbf{w}_{n}$. Since $\mathbf{u}_{1} \mathbf{v}_{1}$ is unitary, we also have:

$$
\begin{aligned}
\rho_{\mathbf{x}_{n}}^{2}\left(\mathbf{w}_{n}\right) & =\rho_{\mathbf{x}_{n}}^{2}\left(\mathbf{u}_{1} \mathbf{v}_{1}\right) \rho_{\mathbf{x}_{n}}^{2}\left(\mathbf{u}_{2} \mathbf{v}_{2} \cdots \mathbf{u}_{k_{n}-1} \mathbf{v}_{k_{n}-1}\right) \\
& =\rho_{\mathbf{x}_{n}}^{2}\left(\mathbf{u}_{1} \mathbf{v}_{1}\right) \widetilde{\mathbf{u}}_{2} \cdots \neq \mathbf{w}_{n} .
\end{aligned}
$$

In the same way, we prove that for all $k<k_{n}$, we have $\rho_{\mathbf{x}_{n}}^{k}\left(\mathbf{w}_{n}\right) \neq \mathbf{w}_{n}$.

In the helix graph of $\widetilde{\mathcal{A}}$ of order $\left(\left|\mathbf{x}_{n}\right|,\left|\mathbf{w}_{n}\right|\right)$, consider the cycle containing the node $\left(\mathbf{x}_{n}, \mathbf{w}_{n}\right)$. Since $\mathbf{w}_{n}$ is unitary, the successors of $\left(\mathbf{x}_{n}, \mathbf{w}_{n}\right)$ on the cycle are: $\left(\mathbf{x}_{n}, \rho_{\mathbf{x}_{n}}\left(\mathbf{w}_{n}\right)\right),\left(\mathbf{x}_{n}, \rho_{\mathbf{x}_{n}}^{2}\left(\mathbf{w}_{n}\right)\right)$, $\ldots$ Therefore the cycle is of length $k_{n}$. Since $k_{n}$ converges to infinity, the lengths of the cycles of the helix graphs of $\widetilde{\mathcal{A}}$ are not uniformly bounded.

\section{Experimentations}

Here, we show how gathering the new criteria with previously known ones allows to decide the (semi)group finiteness for substantially more Mealy automata (at least for those with small alphabet and stateset — of size up to 3).

\footnotetext{
${ }^{1}$ The table shows that 52 out of 76 (isomorphism classes of) 2-letter 2-state Mealy automata can be treated directly using either the old or the new criteria. But actually, the finiteness problem is solved for the 76 cases. Indeed, a series of papers dealing specifically with 2-letter 2-state Mealy automata (see [7] and references therein) has contributed to the actual state of knowledge: 48 automata generate finite semigroups, 10 generate semigroups of linear growth, 17 generate semigroups of exponential growth and 1 generates the semigroup $\mathbf{S}_{\mathbf{I}_{\mathbf{2}}}$ of intermediate growth (see Table 2).
} 
Table 3: Results of experimentations on 2-letter 2-state Mealy automata.

\begin{tabular}{|c|c|c|c|c|c|c|c|c|c|}
\hline & \multirow{2}{*}{\multicolumn{4}{|c|}{ invertible }} & \\
\hline & & & & & & & & & \\
\hline & 2-letter 2-state & i.JIR & JI & JIR & $\overline{\text { BIR }}$ & diJIR & dJI & $\mathbf{N}$ & $\mathbf{W}$ \\
\hline & Mealy automata & 1 & 14 & 1 & 8 & 1 & 14 & 37 & 76 \\
\hline & & & & \multicolumn{4}{|c|}{ reversible } & & \\
\hline \multirow{8}{*}{ 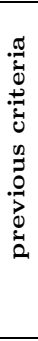 } & Finitary & - & 5 & - & 3 & - & - & 1 & 9 \\
\hline & Thompson-Wielandt & - & - & - & 5 & - & - & - & 5 \\
\hline & Level-transitive & 1 & 4 & 1 & - & - & - & - & 6 \\
\hline & Sidki & - & 1 & - & - & - & - & - & 1 \\
\hline & Limitary cycles & - & 4 & $\overline{-}$ & 6 & $\overline{-}$ & 8 & 6 & 6 \\
\hline & Cayley $^{ \pm}$ & 1 & 1 & 1 & - & $\overline{-}$ & 1 & 2 & 6 \\
\hline & Dual Cayley ${ }^{ \pm}$ & 1 & - & 1 & 1 & - & - & 3 & 6 \\
\hline & union & 1 & 11 & 1 & 8 & - & 8 & 8 & 37 \\
\hline \multirow{6}{*}{ 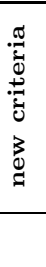 } & $\mathfrak{m o}$-trivial & - & 10 & - & 8 & - & 10 & 11 & 39 \\
\hline & Cycles & 1 & - & 1 & - & - & - & - & 2 \\
\hline & +Sum & - & - & - & 3 & - & 4 & - & 7 \\
\hline & +Dual & - & 8 & 1 & 8 & 1 & 11 & 8 & 37 \\
\hline & union & 1 & 10 & 1 & 8 & 1 & 14 & 13 & 48 \\
\hline & total union & 1 & 14 & 1 & 8 & 1 & 14 & 13 & $52^{1}$ \\
\hline
\end{tabular}

\subsection{Partition}

For convenience of exposition, we introduce the decomposition of the whole class of Mealy automata $\mathbf{W}$ (up to isomorphism) into a disjoint union of seven subclasses. By denoting $\mathbf{I}$ the class of invertible Mealy automata and IR the class of invertible-reversible Mealy automata, the seven classes are defined as follows:

BIR is the class of bireversible Mealy automata,

JIR (standing for Just IR) is the complementary in IR of BIR,

iJIR consists of the inverses of automata from JIR,

diJIR consists of the duals of automata from iJIR,

$\mathbf{J I}$ (standing for $\mathbf{J}$ ust $\mathbf{I}$ ) is the complementary in $\mathbf{I}$ of the union $\mathbf{I R} \cup \mathfrak{i J I R}$,

$\mathfrak{d} \mathbf{J I}$ consists of the duals of automata from $\mathbf{J I}$,

$\mathbf{N}$ is the complementary (in $\mathbf{W}$ ) of the (disjoint) union of the previous six.

\subsection{Previous criteria}

\section{Previously implemented criteria}

The GAP packages FR and automgrp (see 4, 13, 23]) both overload the functions Order and IsFinite by using several criteria mainly coming from geometric group theory. More precisely, we have tested all the corresponding functions: IsFinitaryFRMachine, IsLevelTransitive and ISFINITE_THOMPSONWIELANDT_FR from FR and IsFractal and IsSphericallyTransitive from automgrp. While the first two work perfectly, the last three may not stop. From a practical point of view, IsSphericallyTransitive allows to discriminate too few automata. Now IsLevelTransitive happens to be much slower than IsFractal, so the latter can be advantageously viewed as a preliminary criterion of the former. The first half of the previous criteria part of the following tables expands the performance of these three criteria coming from geometric 
group theory. For 2-letter 3-state and 3-letter 2-state (resp. 3-letter 3-state) automata, the execution time of IsFractal and IsLevelTransitive was limited to $100000 \mathrm{~ms}$ (resp. $200000 \mathrm{~ms}$ ). The resulting data have to be considered with this arbitrary limitation in mind, together with the observation that both functions happen to be significantly sensitive to the representative inside an isomorphism class.

\section{Sidki's criterion}

Based on Sidki's fundamental work, the solution to the order problem [10, 26, for the class of so-called bounded automorphisms — that is, with growth degree at most 0 - may provide an infiniteness criterion: in any invertible automaton $(A, \Sigma, \delta, \rho)$, a bounded state $x \in A$ has infinite order whenever there exists a label $i \mid j$ with $j \neq i \in \Sigma$ on an edge between $x$ and some state belonging to the same strongly connected component. This criterion appears as the second field of the previous criteria part of the tables.

\section{Antonenko's criterion}

An interesting point of view is to investigate those automata $(A, \Sigma, \delta)$ compelling all the Mealy automata $(A, \Sigma, \delta, \rho)$ to generate a finite semigroup. A complete characterization of the latter in term of limitary cycle given in 2] (see also [25]) provides a simple effective criterion for finiteness. An automaton is with limitary cycle whenever every state $x \in A$ accessible from some cyclic one $y \in A$ (that is, there exists a nontrivial word $w \in \Sigma^{*}$ satisfying $\left.\delta_{w}(y)=y\right)$ is without branch (that is, $\delta_{i}(x)=\delta_{j}(x)$ holds for any $(i, j) \in \Sigma^{2}$ ). First considered in [3], the branchless condition alone is covered by Proposition 2.7 and a fortiori by Theorem 3.6. This criterion appears as third field of the previous criteria part of the tables.

\section{Maltcev's criterion}

Let $S$ be a finite semigroup. Define the Cayley machine $C(S)$ (resp. the dual ${ }^{2}$ Cayley machine $\left.C^{*}(S)\right)$ to be the Mealy automaton with stateset $S$, alphabet $S$, and the following transitions: $\forall x, y \in S$,

$$
C(S): \quad x \stackrel{y \mid x y}{\longrightarrow} x y, \quad C^{*}(S): \quad x \stackrel{y \mid y x}{\longrightarrow} x y
$$

According to [21] (see also 22, 12]), for every finite semigroup $S$, the semigroup generated by $C(S)$ (resp. by $C^{*}(S)$ ) is finite if and only if $S$ is $\mathcal{H}$-trivial (resp. $S$ is $\mathcal{H}$-trivial and does not contain non-trivial right zero subsemigroups). This can be viewed as an effective finiteness criterion for those Mealy automata whose isomorphism class intersects the special class of Cayley machines (resp. dual Cayley machines) and their possible inverses (which justifies the symbol \pm in the tables). These two criteria coming from semigroup theory compose the last quarter of the previous criteria part of the tables.

\subsection{New criteria}

The first criterion of the new criteria part is the mo-triviality from Theorem 3.6. Next, the criterion Cycles corresponds to Corollary 4.3 which ensures that every automaton from iJIR and JIR generates an infinite group. The last two criteria are "relative criteria" — which vindicates the symbol + - allowing in good cases to reduce or transpose the finiteness question to smaller and/or simpler automata. The criterion +Sum follows from the easy observation: provided that a Mealy automaton decomposes into a sum of (smaller) Mealy automata, it generates an infinite semigroup whenever one sum component does so. Finally, the criterion +Dual follows from Proposition 2.7.

\footnotetext{
${ }^{2}$ It should be emphasized that the current term dual for a Cayley machine is not consistent with the widely used term dual for a Mealy automaton.
} 
As a simple illustration, let us consider the Mealy automaton $\mathcal{C}$ below on the left. None of the previously known criteria is suitable to detect the infiniteness of $\langle\mathcal{C}\rangle$. Now, the dual $\mathfrak{d}(\mathcal{C})$ happens to be a sum whose 2-state component is (isomorphic to) the dual $\mathfrak{d}(\mathcal{B})$ of the baby Alešin automaton $\mathcal{B}$ (see Table 2), which turns out to be level-transitive.

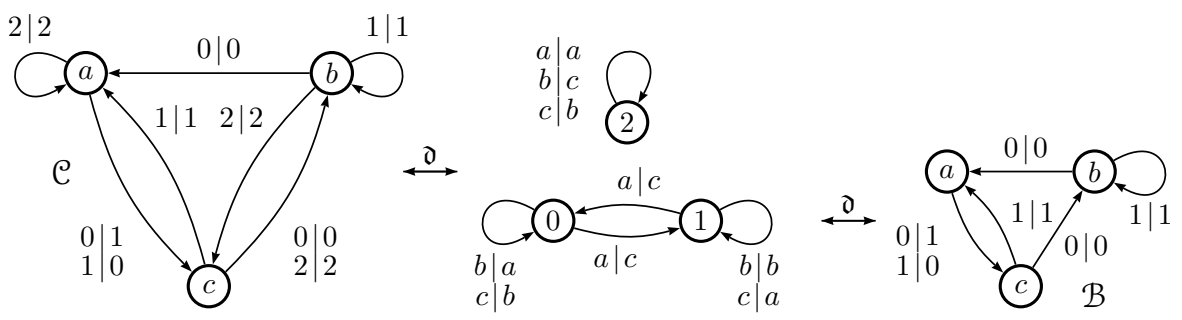

In this way, the isomorphism class of $\mathcal{B}$ contributes for one in the Level-transitive row only, those of $\mathfrak{d}(\mathcal{B})$ and $\mathfrak{d}(\mathcal{C})$ both contribute for one in the respective + Dual rows only and finally that of $\mathcal{C}$ contributes for one in the + Sum row only.

Table 4: Results of experimentations on 2-letter 3-state Mealy automata.

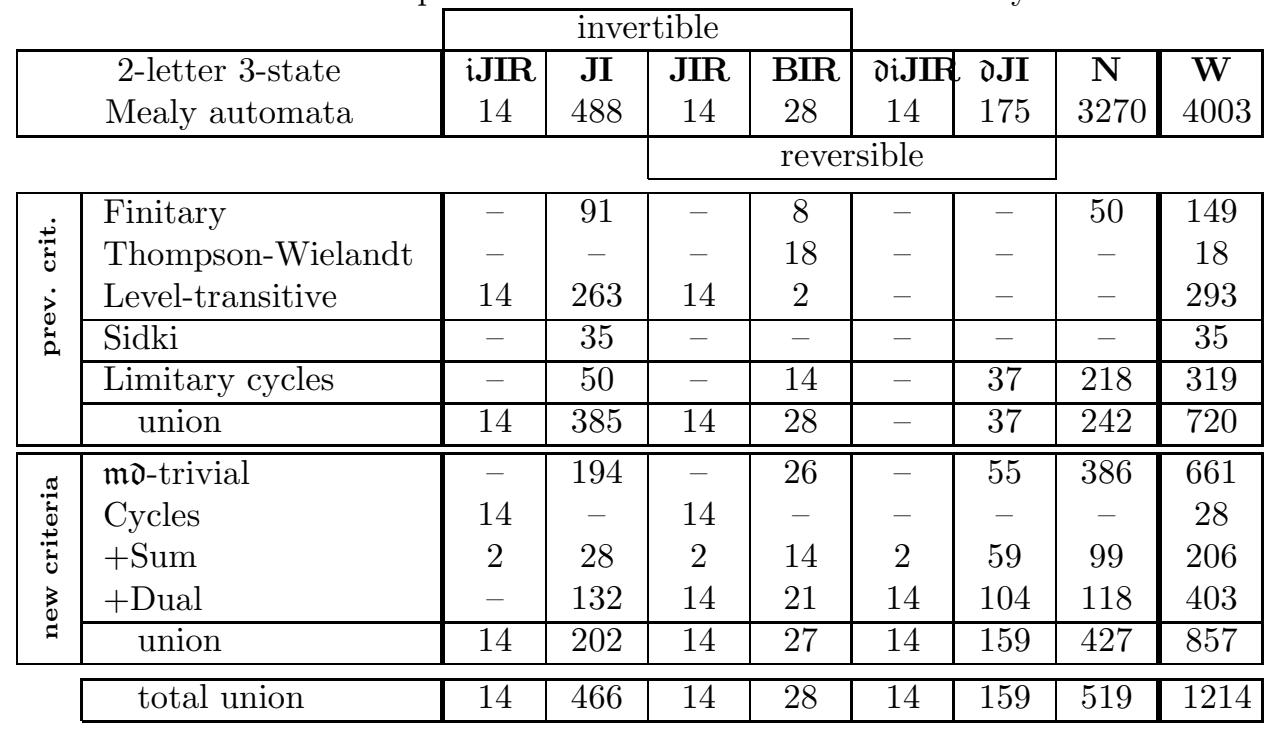

\section{Conclusion}

In this paper, we have emphasized the interest of the duality of Mealy automata for the finiteness problem. Our new approaches enable to treat a much larger number of Mealy automata as before, see Section 5 . We also completely settle the case of non-bireversible IR-automata (they generate infinite groups). On the downside, the decidability of the finiteness problem remains open. However, we believe that the characterization in Theorem 4.4 could lead to a decision procedure for bireversible automata. Indeed, experimentations show that the cycle-lengths stay almost constant for known finite groups and increase extremely fast for known infinite groups. 
Table 5: Results of experimentations on 3-letter 2-state Mealy automata.

\begin{tabular}{|c|c|c|c|c|c|c|c|c|c|}
\hline & \multicolumn{4}{|c|}{ invertible } & \\
\hline \multicolumn{2}{|c|}{ 3-letter 2-state } & iJIR & $\mathbf{J I}$ & JIR & BIR & diJIR & dJI & $\mathbf{N}$ & $\bar{W}$ \\
\hline \multicolumn{2}{|r|}{ Mealy automata } & 14 & 175 & 14 & 28 & 14 & 488 & 3270 & 4003 \\
\hline & & \multicolumn{6}{|c|}{ reversible } & \\
\hline \multirow{6}{*}{ 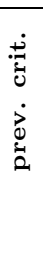 } & Finitary & - & 11 & - & 4 & - & - & 4 & 19 \\
\hline & Thompson-Wielandt & - & - & - & 13 & - & - & - & 13 \\
\hline & Level-transitive & 11 & 84 & 12 & - & - & - & - & 107 \\
\hline & Sidki & $\overline{-}$ & 2 & - & $=$ & $\overline{-}$ & $\overline{-}$ & $\overline{-}$ & 2 \\
\hline & Limitary cycles & - & 11 & - & 16 & - & 132 & 118 & 277 \\
\hline & partial union & 11 & 104 & 12 & 21 & $\overline{-}$ & 132 & 118 & 398 \\
\hline \multirow{6}{*}{ 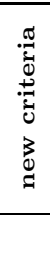 } & $\overline{\mathfrak{m} \mathfrak{d} \text {-trivial }}$ & $\overline{-}$ & $\overline{55}$ & $\overline{-}$ & 26 & $\overline{-}$ & $\overline{194}$ & 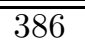 & 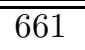 \\
\hline & Cycles & 14 & - & 14 & - & - & - & - & 28 \\
\hline & + Sum & - & - & - & 8 & - & 66 & - & 74 \\
\hline & + Dual & 2 & 69 & 14 & 28 & 14 & 395 & 313 & 835 \\
\hline & partial union & 14 & 75 & 14 & 28 & 14 & 466 & 519 & 1130 \\
\hline & total union & $\overline{14}$ & $\overline{159}$ & $\overline{14}$ & $\overline{28}$ & $\overline{14}$ & $\overline{466}$ & 519 & $\overline{1214}$ \\
\hline
\end{tabular}

\section{References}

[1] S. V. Alešin. Finite automata and the Burnside problem for periodic groups. Mat. Zametki, 11:319-328, 1972.

[2] A. S. Antonenko. On transition functions of Mealy automata of finite growth. Matematychni Studii., 29(1):3-17, 2008.

[3] A. S. Antonenko and Berkovich E. L. Groups and semigroups defined by some classes of Mealy automata. Acta Cybernetica, 18(1):23-46, 2007.

[4] L. Bartholdi. FR Functionally recursive groups, Self-similar groups - a GAP package, Version 1.1.3, 2010.

[5] L. Bartholdi, R. I. Grigorchuk, and V. Nekrashevych. From fractal groups to fractal sets. In Fractals in Graz 2001, Trends Math., pages 25-118. Birkhäuser, 2003.

[6] L. Bartholdi, R. I. Grigorchuk, and Z. Šuniḱ. Branch groups. In Handbook of algebra, Vol. 3, pages 989-1112. North-Holland, 2003.

[7] L. Bartholdi, I. I. Reznykov, and V. I. Sushchanskiǔ. The smallest Mealy automaton of intermediate growth. J. Algebra, 295(2):387-414, 2006.

[8] L. Bartholdi and P. V. Silva. Groups defined by automata. 2010. "arXiv:cs.FL/1012.1531".

[9] I. Bondarenko, R. I. Grigorchuk, R. Kravchenko, Y. Muntyan, V. Nekrashevych, D. Savchuk, and Z. Šunić. On classification of groups generated by 3-state automata over a 2-letter alphabet. Algebra Discrete Math., (1):1-163, 2008.

[10] I. V. Bondarenko, Bondarenko N. V., Sidki S. N., and Zapata F. R. On the conjugacy problem for finite-state automorphisms of regular rooted trees. arXiv:math.GR/1011.2227.

[11] M. Burger and S. Mozes. Groups acting on trees: from local to global structure. Inst. Hautes Études Sci. Publ. Math., (92):113-150, 2000.

[12] A. J. Cain. Automaton semigroups. Theor. Comput. Sci., 410:5022-5038, 2009.

[13] The GAP Group. GAP - Groups, Algorithms, and Programming, Version 4.4.12, 2008. 
Table 6: Results of experimentations on 3-letter 3-state invertible or reversible automata.

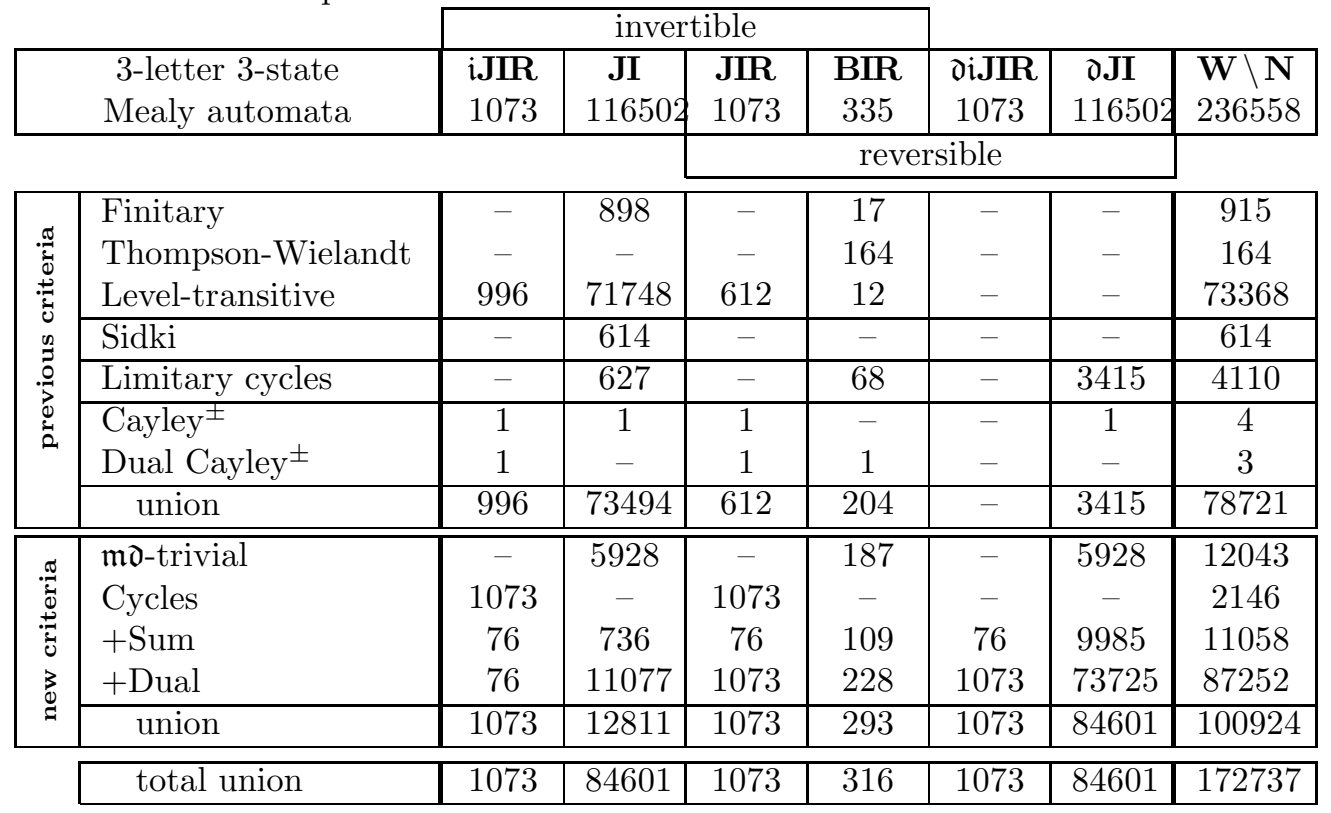

[14] Y. Glasner and S. Mozes. Automata and Square Complexes. Geom. Dedicata, 111(1):43-64, 2005.

[15] R. Grigorchuk. Semigroups with cancellations of degree growth. Mat. Zametki, 43:305-319, 1988.

[16] R. I. Grigorchuk. On Burnside's problem on periodic groups. Funktsional. Anal. i Prilozhen., 14(1):53-54, 1980.

[17] R. I. Grigorchuk. On the Milnor problem of group growth. Dokl. Akad. Nauk SSSR, 271(1):30$33,1983$.

[18] R. I. Grigorchuk, V. V. Nekrashevich, and V. I. Sushchanskiǔ. Automata, dynamical systems, and groups. Tr. Mat. Inst. Steklova, 231:134-214, 2000.

[19] R. I. Grigorchuk and Z. Šnić. Self-similarity and branching in group theory. In Groups St. Andrews 2005. Vol. 1, volume 339 of London Math. Soc. Lecture Note Ser., pages 36-95. Cambridge Univ. Press, 2007.

[20] O. Macedońska, V. Nekrashevych, and V. I. Sushchanskiı̌. Commensurators of groups and reversible automata. Dopov. Nats. Akad. Nauk Ukr. Mat. Prirodozn. Tekh. Nauki, (12):36-39, 2000.

[21] V. Maltcev. Cayley automaton semigroups. Internat. J. Algebra Comput., 19(1):79-95, 2009.

[22] A. Mintz. On the Cayley semigroup of a finite aperiodic semigroup. Internat. J. Algebra Comput., 19(6):723-746, 2009.

[23] Y. Muntyan and D. Savchuk. automgrp Automata Groups - a GAP package, Version 1.1.4.1, 2008.

[24] V. Nekrashevych. Self-similar groups, volume 117 of Mathematical Surveys and Monographs. American Mathematical Society, Providence, RI, 2005.

[25] A. Russyev. Finite groups as groups of automata with no cycles with exit. Algebra and Discrete Mathematics, 9(1):86-102, 2010. 
[26] S. N. Sidki. Automorphisms of one-rooted trees: growth, circuit structure, and acyclicity. J. Math. Sci. (New York), 100(1):1925-1943, 2000. Algebra, 12.

[27] P. V. Silva and B. Steinberg. On a class of automata groups generalizing lamplighter groups. Internat. J. Algebra Comput., 15(5-6):1213-1234, 2005.

[28] B. Steinberg, M. Vorobets, and Y. Vorobets. Automata over a binary alphabet generating free groups of even rank. Internat. J. Algebra Comput., 2006. "arXiv:math.GR/0610033".

[29] Z. Sunik and E. Ventura. The conjugacy problem is not solvable in automaton groups. 2010. arXiv:math.GR/1010.1993.

[30] M. Vaughan-Lee. The restricted Burnside problem, volume 8 of London Mathematical Society Monographs. New Series. Oxford University Press, 1993.

[31] E. I. Zel'manov. Solution of the restricted Burnside problem for groups of odd exponent. Izv. AN SSSR Math+, 54(1):42-59, 221, 1990.

[32] E. I. Zel'manov. Solution of the restricted Burnside problem for 2-groups. Mat. Sb., 182(4):568-592, 1991. 